MS8. Molecular machines and motors

\section{MS8-01 Transporting cargo over long distances: insight from dynein/dynactin structures}

Andrew Carter $^{1}$

1. MRC Laboratory of Molecular Biology, Francis Crick Ave, Cambridge, CB2 0QH, UK

email: cartera@mrc-lmb.cam.ac.uk

Cells depend on components being moved to the correct place at the correct time. My group is interested in cytoplasmic dynein-1 (dynein-1), a motor which delivers many different cargos via the microtubule network. When dynein-1 is mutated it leads to neurodegeneration and it is susceptible to hijack by viruses which use it to travel into the cell. We have determined X-ray crystal structures of the dynein motor before and after it binds and hydrolyses ATP. We subsequently used single molecule fluorescence assays to show how a cofactor, dynactin, activates the full length 1.4MDa dynein complex to move long distances

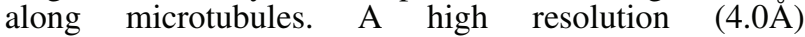
cryo-electron microscopy (cryo-EM) structure of dynactin explained how this 23 subunit complex is assembled. We also used a combination of cryo-EM and $\mathrm{X}$-ray crystallography to show how dynactin binds to dynein. The two complexes are only brought together in the presence of an adaptor protein, Bicaudal-D2, that links them to the cargo they will carry. This suggests the large and intricate dynein/dynactin transport machine only assembles when a cargo is ready to move.

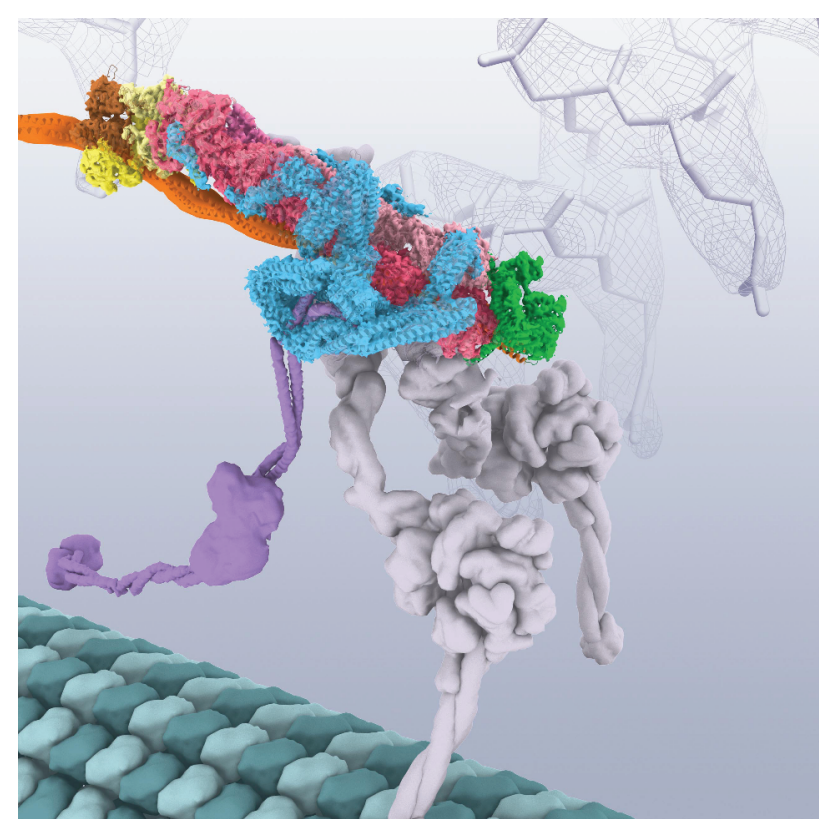

Figure 1. Model of the structure of cytoplasmic dynein (grey) bound to dynactin (multicolor) via the Golgi vesicle cargo adaptor BICD2 (orange). Dynein is reaching down towards its microtubule track.

Keywords: dynein, microtubule, motor, AAA+, machine, actin 